NOVEMBER 6, 2018

Preprint typeset using $\mathrm{LT}_{\mathrm{E}} \mathrm{X}$ style emulateapj v. 12/14/05

\title{
THE EVOLUTION OF SPIRAL, S0 AND ELLIPTICAL GALAXIES IN CLUSTERS
}

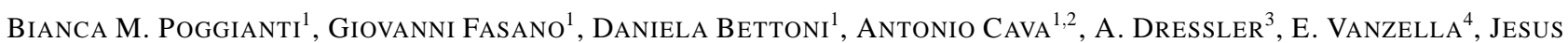

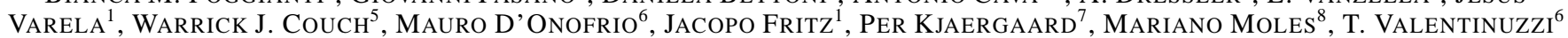 \\ ${ }^{1}$ INAF-Astronomical Observatory of Padova, Italy, ${ }^{2}$ Instituto de Astrofisica de Canarias La Laguna, Spain, ${ }^{3}$ The Observatories of the Carnegie institution of \\ Washington, Pasadena, USA, ${ }^{4}$ INAF-Astronomical Observatory of Trieste, Italy ${ }^{5}$ Center for Astrophysics and Supercomputing, Swinburne University of \\ Technology, Australia ${ }^{6}$ Astronomical Department, University of Padova, Italy, ${ }^{7}$ Copenhagen University Observatory. The Niels Bohr Institute for Astronomy, \\ Physics and Geophysics, Denmark, ${ }^{8}$ Instituto de Astrofisica de Andalucia, CSIC, Granada, Spain \\ November 6, 2018
}

\begin{abstract}
We quantify the evolution of the spiral, S0 and elliptical fractions in galaxy clusters as a function of cluster velocity dispersion $(\sigma)$ and X-ray luminosity $\left(L_{X}\right)$ using a new database of 72 nearby clusters from the WIdeField Nearby Galaxy-cluster Survey (WINGS) combined with literature data at $z=0.5-1.2$. Most WINGS clusters have $\sigma$ between 500 and $1100 \mathrm{~km} \mathrm{~s}^{-1}$, and $L_{X}$ between 0.2 and $5 \times 10^{44} \mathrm{erg} / \mathrm{s}$. The S0 fraction in clusters is known to increase with time at the expense of the spiral population. We find that the spiral and S0 fractions have evolved more strongly in lower $\sigma$, less massive clusters, while we confirm that the proportion of ellipticals has remained unchanged. Our results demonstrate that morphological evolution since $z=1$ is not confined to massive clusters, but is actually more pronounced in low mass clusters, and therefore must originate either from secular (intrinsic) evolution and/or from environmental mechanisms that act preferentially in lowmass environments, or both in low- and high-mass systems. We also find that the evolution of the spiral fraction perfectly mirrors the evolution of the fraction of star-forming galaxies. Interestingly, at low-z the spiral fraction anticorrelates with $L_{X}$. Conversely, no correlation is observed with $\sigma$. Given that both $\sigma$ and $L_{X}$ are tracers of the cluster mass, these results pose a challenge for current scenarios of morphological evolution in clusters.

Subject headings: galaxies: clusters: general — galaxies: evolution — galaxies: structure — galaxies: fundamental parameters
\end{abstract}

\section{INTRODUCTION}

The strongest evidence that a significant fraction of today's early-type galaxies have evolved from later-type galaxies at relatively recent cosmic epochs comes from galaxy cluster studies. HST images revealed that spirals are proportionally much more common, and S0 galaxies much rarer, in distant than in nearby clusters, suggesting that many of the local cluster S0s have evolved from spirals (Dressler et al. 1997, Fasano et al. 2000, Treu et al. 2003, Postman et al. 2005, Smith et al. 2005, Desai et al. 2007, but see Andreon 1998 and Holden et al. 2009 for an opposite view). While the paucity of the S0 population has been observed out to $z \sim 1$, it seems that most of the morphological evolution occurs at $z \leq 0.4$, because the S0 and spiral galaxy fractions flatten out in clusters at $z>0.45$ (Desai et al. 2007). Interestingly, no significant evolution has been detected in the proportion of cluster ellipticals since $z \sim 1$ (see references above).

The latest high-z studies of galaxy morphologies in clusters have started to assess how the morphological mix depends on the global cluster properties, in particular on $L_{X}$ and $\sigma$, both considered rather good tracers of the cluster mass (Postman et al. 2005, Desai et al. 2007). Early studies at $z \sim 0$ had found that the proportions of spirals and early-type galaxies vary with $L_{X}$ (Bahcall 1977, McHardy 1978, Edge \& Stewart 1991). Clearly, it is necessary to quantify the morphological evolution as a function of cluster mass, in order to perform a meaningful comparison between high- and low-z data and to investigate the origin of the morphological variations with global environment.

To date, a quantitative analysis of the morphological evolution for clusters of different masses has been impossible, due to the lack of a local large sample of clusters with similarly detailed informations as the high-z samples. In this Letter, we present the comparison between a new dataset of 72 clusters in the local universe and the distant clusters currently available in the literature, quantifying the evolution of the fractions of spiral, S0 and elliptical galaxies in clusters with a wide range of velocity dispersions and $L_{X}$.

Throughout this paper we use $\left(H_{0}, \Omega_{m}, \Omega_{\lambda}\right)=(70,0.3,0.7)$.

\section{OBSERVATIONS}

The WIde-Field Nearby Galaxy-cluster Survey (WINGS) is a multiwavelength photometric and spectroscopic survey of 77 galaxy clusters at $0.04<z<0.07$ (Fasano et al. 2006). Clusters were selected in the X-ray from the ROSAT Brightest Cluster Sample and its extension (Ebeling et al. 1998, 2000) and the X-ray Brightest Abell-type Cluster sample (Ebeling et al. 1996). ${ }^{1}$ WINGS clusters cover a wide range of $\sigma$, typically between 500 and $1100 \mathrm{~km} \mathrm{~s}^{-1}$, and $L_{X}$, typically $0.2-5 \times$ $10^{44} \mathrm{erg} / \mathrm{s}$.

WINGS has obtained wide-field optical photometry $(B V)$ for all 77 fields (Fasano et al. 2006, Varela et al. 2009), as well as infrared $(J K)$ photometry (Valentinuzzi et al. 2009), optical spectroscopy (Cava et al. 2009), U band and $\mathrm{H} \alpha$ narrow-band imaging for a subset of the WINGS clusters. In this Letter we consider 72 WINGS clusters, having excluded only clusters with no reliable velocity dispersion measurement (A311, A2665, A3164 and Z1261) or insufficient $V$-band seeing quality (A3562). Hereafter, we use galaxy $V$ total magnitudes (Sextractor MAGAUTO) from Varela et al. (2009). Cluster velocity dispersions were computed combining WINGS and literature redshifts. For all but one cluster, they are based on more than 20 spectroscopic members, with an average of 92 spectroscopic members per cluster (Cava et al. 2009).

\footnotetext{
${ }^{1}$ X-ray luminosities (0.1-2.4keV) from Ebeling et al. (1996, 1998, 2000) have been converted to the cosmology used in this paper.
} 
Our analysis is based on galaxy morphological types, derived from $V$ images using the purposely devised tool MORPHOT (Fasano et al. 2009, in preparation). Our approach is a generalization of the non-parametric method proposed by Conselice et al. (2000), see also Conselice (2003). In particular, we have extended the classical CAS (Concentration/Asymmetry/clumpinesS) parameter set by introducing a number of additional, suitably devised morphological indicators, using a final set of 10 parameters. A control sample of $\sim 1000$ visually classified galaxies has been used to calibrate the whole set of morphological indicators, with the aim of identifying the best sub-set among them, as well as of analyzing how they depend on galaxy size, flattening and $\mathrm{S} / \mathrm{N}$ ratio.

The morphological indicators have been combined with two independent methods, a Maximum Likelihood analysis and a Neural Network trained on the control sample of visually classified galaxies. The final, automatic morphological classification combines the results of both methods. It has been carefully verified that our morphological classifications reproduce visual classifications by two of us with a robustness and a reliability comparable with those obtained from visual inspection by experienced independent classifiers (Fasano et al. 2009 , in prep.).

In the following, we use three broad morphological classes, ellipticals, S0s and "spirals", where the spiral class includes any galaxy later than an S0.

As high-z comparison, we use the morphological fractions of the ESO Distant Cluster Survey (EDisCS) sample of 10 clusters at $z=0.5-0.8$ from Desai et al. (2007), and the 5 clusters at $z=0.8-1.2$ from Postman et al. (2005) for which a $\sigma$ is available. ${ }^{2}$ The morphological classification of galaxies in these clusters is based on visual classification of HST/ACS F814W images sampling the rest-frame $\sim 4300-5500$ range. The reliability of the high- $\mathrm{z}$ morphologies have been tested and discussed at length by Postman et al. (2005) and Desai et al. (2007), and by previous works. For the high-z clusters we use the X-ray luminosities in the $0.5-2 \mathrm{keV}$ band provided by Olivia Johnson (2008 private communication) and from Johnson et al. (2006) and Postman et al. (2005).

The analysis for both WINGS and high-z clusters includes only galaxies within $0.6 R_{200}{ }^{3}$ brighter than $M_{V}=-19.5$ for WINGS ${ }^{4}$ and $M_{V}=-20$ for EDisCS. The different magnitude limit accounts for passive evolution dimming between the mean WINGS redshift and $z \sim 0.6$. The fractions taken from Postman et al. (2005) already take passive evolution into account, corresponding to $M_{V}=-19.3$ at $z=0$. High$\mathrm{z}$ morphological fractions are corrected for fore-background contamination by the authors. For WINGS clusters, we have verified that the field contamination is entirely negligible using the local field counts and morphological mix by Whitmore et al. (1993).

This is the first time the morphological evolution in clusters can be investigated as a function of a proxy for the clus-

\footnotetext{
${ }^{2}$ For the $\mathrm{Cl} 1604+4304$ and $\mathrm{Cl} 1604+4321$ clusters we have used revised $1.0 h_{70}^{-1} \mathrm{Mpc}$ velocity dispersions from Gal et al. 2008.

${ }^{3} R_{200}$ is defined as the radius delimiting a sphere with interior mean density 200 times the critical density, approximately equal to the cluster virial radius. $0.6 R_{200}$ roughly corresponds to $R_{500}$, whose interior mean density is 500 times the critical density. The variation of the morphological fractions between 0.6 and $1 R_{200}$ in Postman et al. (2005) is negligible (their Table 3). A few WINGS clusters have photometric coverage slightly smaller than $0.6 R_{200}$ (Cava et al. 2009). None of the results in this paper change if these clusters are excluded from the analysis.

${ }^{4} 63 \%$ of the galaxies fulfilling these criteria are spectroscopically confirmed members.
}

TABLE 1

AVERAGE MORPHOLOGICAL FRACTIONS.

\begin{tabular}{lccc}
\hline & \multicolumn{2}{c}{ High-z } & Low-z \\
\hline & Low- $\sigma$ & High- $\sigma$ & All- $\sigma$ \\
\hline Spirals & 60 & 40 & $\mathbf{2 3}$ \\
S0s & 15 & 22 & $\mathbf{4 4}$ \\
Ellipticals & 25 & 37 & $\mathbf{3 3}$ \\
\hline
\end{tabular}

NOTE. - Low- $\sigma$ and high- $\sigma$ clusters are those with $\sigma=500-700 \mathrm{~km} \mathrm{~s}^{-1}$ and $>700 \mathrm{~km} \mathrm{~s}^{-1}$, respectively. At low redshift, the average fractions are constant with velocity dispersion (see text).

ter mass, such as velocity dispersion or X-ray luminosity. This is possible thanks to the combination of the first highz (EDisCS) and first low-z (WINGS) homogeneous samples with simultaneously a large range in cluster masses, detailed galaxy morphologies and accurate velocity dispersions.

\section{RESULTS: THE EVOLUTION WITH REDSHIFT OF THE MORPHOLOGICAL MIX}

Figure 1 shows the fractions of spiral, S0, elliptical and early-type $(\mathrm{E}+\mathrm{S} 0)$ galaxies as a function of cluster velocity dispersion.

At low-z (black points), none of the morphological fractions show a trend with velocity dispersion. Spearman probabilities of a correlation are lower than $70 \%$ in all cases, and least square fits yield a practically flat best fit. On average, spirals are $23 \% \pm 9 \%$ of all galaxies, S0 galaxies represent almost half of the cluster population $(44 \% \pm 10 \%)$ and ellipticals are about a third with an average fraction of $33 \% \pm 7$. The quoted errors on these average values correspond to the rms, and represent the cluster-to-cluster scatter in the morphological fractions at a given $\sigma .^{5}$ For all morphological types, this cluster-to-cluster scatter is comparable or even smaller than the average of all poissonian errorbars on the morphological fractions of individual clusters (Fig. 1), indicating a quite remarkable homogeneity of the morphological mix in nearby clusters, regardless of velocity dispersion, over a large range of $\sigma$.

In contrast, mild correlations both with $\sigma$ and $L_{X}$ have been reported in distant clusters by Postman et al. (2005) and Desai et al. (2007) (see also Figs. 1 and 2). Comparing with clusters at $z=0.5-1.2$ (red points in Fig. 1), we find that the morphological evolution has been much stronger in progressively lower velocity dispersion systems. As shown in Fig. 1 and summarized in Table 1, on average, the spiral fraction has evolved from $\sim 60 \%$ for systems with $\sigma=500-700 \mathrm{~km} \mathrm{~s}^{-1}$, and from $\sim 40 \%$ for more massive clusters, to $\sim 23 \%$ at low-Z regardless of velocity dispersion. ${ }^{6}$ Similarly, the average S0 fraction has changed from $\sim 15 \%$ for the least massive clusters, and from $\sim 22 \%$ for the most massive clusters, to $44 \%$ in nearby clusters of all $\sigma$ 's. Finally, the evolution of the average elliptical fraction for massive clusters is negligible, as noted by previous works (eg. Dressler et al. 1997).

\subsection{Comparison with the evolution of the star-forming fraction}

\footnotetext{
${ }^{5}$ We note that the most massive clusters with $\sigma \geq 1000 \mathrm{~km} \mathrm{~s}^{-1}$ possibly display a lower scatter than lower $\sigma$ systems, and tend to have among the lowest spiral fractions, and highest eraly-type fractions, but the number of very massive clusters is too low to draw robust conclusions.

6 Since the morphological fractions are flat with $\sigma$ at low redshift, the evolution of the cluster $\sigma$ 's with redshift is irrelevant when assessing the morphological evolution.
} 
We now compare the evolution of the morphological fractions with the evolution of the fraction of star-forming galaxies. The star-forming fraction as a function of cluster $\sigma$ was derived as the proportion of [OII]-line emitters, defined to have an $[\mathrm{OII}]$ equivalent width $\geq 3 \AA$, for the EDisCS sample and for an SDSS nearby cluster sample by Poggianti et al. (2006), for the same galaxy magnitude limits used here and within $R_{200}$. The best fit trends of star-forming fractions at high- and low-z (blue lines) are reproduced and compared to the spiral fractions in the leftmost panel of Fig. 1 (see also the rightmost panel for the complementary passively evolving (=non-emission-line) versus early type comparison).

The qualitative and, roughly speaking, also quantitative correspondence between spiral and star-forming fractions (and, equivalently, early-type and passively evolving fractions) is striking both for nearby and distant clusters. It is well known that not all early-type galaxies are passively evolving and not all spirals are star-forming, as testified by the (small) number of ellipticals and SOs with emission lines and the (significant) number of passive spirals observed in cluster spectroscopic surveys (eg. Dressler et al. 1999, Sanchez-Blazquez et al. 2009). However, the agreement in Fig. 1 indicates that, overall, the morphological evolution and the evolution in the proportion of star-forming galaxies have been very similar. In spite of the evidence that morphological transformations occur on a longer timescale than star formation changes (Poggianti et al. 1999), this agreement suggests that a common physical cause is likely to regulate both on the long run. In this respect, it is interesting to note that "morphology" is more strongly affected by star formation, and depends more strongly on environment, than "galaxy structure" as measured for example by the Sersic index, that mainly depends on galaxy mass (van der Wel 2008).

\subsection{Trends with $X$-ray luminosity}

Finally, we analyze the morphological trends as a function of $L_{X}$ (Fig. 2). The morphological evolution is similar to that observed for $\sigma$ : at a given $L_{X}$, distant clusters have higher spiral fractions, and lower S0 fractions, than local clusters. Elliptical fractions are comparable at high- and low-z.

Interestingly, in contrast with the lack of correlation with $\sigma$, and in spite of the fact that both $\sigma$ and $L_{X}$ are considered tracers of cluster mass, we find that the spiral fraction is statistically anticorrelated with $L_{X}$, with a Spearman probability of $99.6 \%$ (Fig. 2). The best fit linear relation and its error computed using bootstrap resampling is $f_{s p}=$ $-0.077 \pm 0.026 \log L_{X}+3.62 \pm 1.15$.

This confirms previous results that found similar trends between morphological fractions and $L_{X}$ (Bahcall 1977, McHardy 1978, Edge \& Stewart 1991). We note that our correlations are less steep with $L_{X}$ than in these previous works, possibly due to the fact that we limit our analysis to a fixed fraction of $R_{200}$, while previous studies included all galaxies within a plate field-of-view.

\section{DISCUSSION AND CONCLUSIONS}

The results presented in this Letter highlight two main issues that are central to understanding why galaxy morphologies change.

First, our findings demonstrate that morphological evolution does not occur exclusively in massive clusters, being actually more conspicuous in low mass clusters. This can be due either to "secular" galaxy evolution (via processes intrinsic to each galaxy), or to one or more environmental processes that work preferentially in low-mass systems, or both in low- and high-mass systems.

Finding correlations of galaxy properties with global parameters like cluster mass, velocity dispersion or X-ray luminosity does not necessarily imply that environment at the epoch of observation is the agent of galaxy evolution. The principal cause could be 'secular,' by which we mean that the evolution of morphology or star formation rests on some fundamental galaxy property/ies, such as galaxy mass, or galaxy matter density at such an early epoch as to be considered "initial conditions." For example, the epoch at which star formation will end and the galaxy's morphology evolve the galaxy's internal 'clock' - could be driven by the primordial density and mass of available gas, conditions that also correlate with the properties of the future global environment, such as the cluster $\sigma$ that we have observed. In other words, initial conditions (the "primordial environment") would predetermine individual galaxy properties, in a statistical sense. Trends with global environments as those we observe would arise because the distribution of initial conditions varies systematically with global environmental properties, such as cluster mass.

If, instead, environmental effects are directly inducing the morphological change close to the time we observe it, our results show that the processes must work effectively either in both low-mass and high-mass clusters, or preferentially in the low-mass cluster range of our sample, and/or perhaps also in groups with even lower masses than those considered here. In the case of environmental effects, the evolution of morphological fractions depends on $\sigma$ because the average cluster growth history (the history of accretion of other clusters, groups and isolated galaxies onto a cluster) varies systematically with cluster mass (Poggianti et al. 2006).

Clearly, the constancy of the spiral, S0 and elliptical fractions with $\sigma$ observed in WINGS at the present epoch cannot continue to systems of even lower velocity dispersions all the way down to isolated galaxies, otherwise the morphological mix of the general field at low-z should be the same as in clusters, whereas it is markedly different. Somewhere below $\sigma=500 \mathrm{~km} \mathrm{~s}^{-1}$ the average fraction of spirals must break or slowly transition to the high fraction of spirals found in the low-density "field" population. Understanding the minimum cluster/group mass at which morphological transformations regularly take place is of course fundamental not only to identifying the relevant mechanisms at work, but also to assessing how many galaxies can be affected and how this fits in with the general cosmic evolution from star-forming/late-type to passive/early-type galaxies; for example, van der Wel. et al. 2007 find no significant evolution in the early-type galaxy fraction among field galaxies.

In this respect, it is important to note that the limit of the WINGS survey, $\sigma \sim 500 \mathrm{~km} \mathrm{~s}^{-1}$, corresponds to the observed break in the trend of star-forming fraction versus $\sigma$ in SDSS clusters at $z=0$ : above $500 \mathrm{~km} \mathrm{~s}^{-1}$ the fraction is flat with $\sigma$ (blue line in Fig. 1), while below the scatter becomes very large, with the average fraction possibly increasing towards lower velocity dispersions (see Figs. 4 and 6 in Poggianti et al. 2006). It is reasonable to expect that the morphological fractions in low $\sigma$ systems continue to follow the trends of the star-forming fraction (as they do above $500 \mathrm{~km} \mathrm{~s}^{-1}$ ), and therefore to expect a large scatter and an increasing average spiral fraction beginning just below $500 \mathrm{~km} \mathrm{~s}^{-1}$. If this is the case, and if the evolution is not secularly, but environmentally, driven, then some process is probably affecting galaxies 
in some, but apparently not all, groups. This is consistent with the fact that, at all redshifts, groups are a very heterogeneous population, some having galaxy properties resembling massive clusters - low spiral and star-forming fractions, with others resembling the field (P06, Jeltema et al. 2007, Wilman et al. 2005, Poggianti et al. 2008, Kautsch et al. 2008, Wilman et al. 2009, Poggianti et al. 2009).

In conclusion, our result suggests that the candidate environmental mechanism(s) should be one (those) that act efficiently and quite universally in clusters with $\sigma \geq 500 \mathrm{~km} \mathrm{~s}^{-1}$, and probably in some, but not all, lower-mass clusters and groups. ${ }^{7}$ Neither tidal effects, nor processes related to the intra-cluster medium, can be easily ruled out at this stage (see Poggianti et al. (2009) and Boselli \& Gavazzi (2006) for a discussion of the possible mechanisms).

The second challenge for any acceptable evolutionary scenario is posed by the existence and lack, respectively, of a dependence of the spiral fraction on $L_{X}$ and $\sigma$ in local clusters, described above. $L_{X}$ and $\sigma$ are rather well correlated for all local cluster samples including WINGS (Cava et al. 2009), and both are considered rather good tracers of cluster mass, albeit with caveats. It is therefore surprising that the morphological distribution correlates at the $3 \sigma$ level with $L_{X}$, but not

\footnotetext{
7 The hypothesis that late-type galaxies suffer a significant morphological change in all systems with masses much lower than $500 \mathrm{kms}^{-1} \sim 10^{14} \mathrm{M}_{\odot}$ can be ruled out by the fact that the early-type/passive fraction in local clus-
}

with $\sigma$. Whether this is due to the way $L_{X}$ and $\sigma$ differently trace the cluster mass, whether it is pointing to some residual trend with the properties of the intra-cluster medium $\left(L_{X}\right)$ on top of a more general evolution, or whether this is support for a secular mechanism where the correlation of properties does not imply a causal connection, is an open question.

We would like to thank the anonymous referee for the constructive and thought-stimulating report. We warmly thank Olivia Johnson for kindly computing the $0.5-2 \mathrm{keV}$ EDisCS luminosities for us, and Vandana Desai for sending the high-z fractions in electronic form. BMP acknowledges the Alexander von Humboldt Foundation and the Max Planck Instituut fur Extraterrestrische Physik in Garching for a very pleasant and productive staying during which the work presented in this paper was carried out. BMP acknowledges financial support from the FIRB scheme of the Italian Ministry of Education, University and Research (RBAU018Y7E) and from the INAF-National Institute for Astrophysics through its PRININAF2006 scheme.

Facilities: $\quad$ INT(WFC), $2.2 \mathrm{~m}(\mathrm{WFC}), \quad$ AAT(2dF), WHT(WYFFOS).

ters should then be much lower than observed (Fig. 16 in Poggianti et al. 2006).

REFERENCES

Andreon, S., 1998, ApJ, 501, 533

Bahcall, N.A., 1977, ApJ, 218, L93

Boselli, A., Gavazzi, G., 2006, PASP, 118, 517

Cava, A., et al., 2009, A\&A in press (astro-ph 0812.2022)

Conselice, C.J., Bershady, M.A., Jangren, A., 2000, ApJ, 529, 886

Conselice, C.J., 2003, ApJS, 147, 1

Desai, V., et al., 2007, ApJ, 660, 1151

Dressler, A., Oemler, A., Couch, W.J., Smail, I., Ellis, R.S., Barger, A., Butcher, H., Poggianti, B.M., Sharples, R.M., 1997, ApJ, 490, 577

Dressler, A., Smail, I., Poggianti, B. M., Butcher, H., Couch, W. J., et al. 1999, ApJS, 122, 51

Ebeling, H., Voges, W., Bohringer, H., Edge, A.C., Huchra, J.P., Briel, U.G., 1996, MNRAS, 281, 799

Ebeling, H., Edge, A.C., Bohringer, H., Allen, S.W., Fabian, A.C., Voges, W., Huchra, J.P., 1998, MNRAS, 301, 881

Ebeling, H., Edge, A.C., Allen, S.W., Crawford, C.S., Fabian, A.C., Huchra, J.P., 2000, MNRAS, 318, 333

Edge, A.C., Stewart, G.C., 1991, MNRAS, 252, 428

Fasano, G., Poggianti, B. M., Couch, W. J., Bettoni, D., et al. 2000, ApJ, 542 , 673

Fasano, G., et al., 2006, A\&A, 445, 805

Gal, R.R., Lemaux, B.C., Lubin, L.M., Kocevski, D., Squires, G.K., 2008, ApJ, 684, 933

Holden, B.P. et al., 2009, ApJ, 693, 617

Jeltema, T.E., Mulchaey, J.S., Lubin, L.M., Fassnacht, C.D., 2007, ApJ, 658, 865

Johnson, O., et al. 2006, MNRAS, 371, 1777
Kautsch, S.J., Gonzales, A.H., Soto, C.A., Tran, K.-V., Zaritsky, D., Moustakas, J., 2008, ApJL, 688, L5

McHardy, I., 1978, MNRAS, 184, 783

Poggianti, B. M., Smail, I., Dressler, A., Couch, W. J., Barger, A. J., Butcher, H., et al. 1999, ApJ, 518, 576

Poggianti, B.M., et al., 2006, ApJ, 642, 188

Poggianti, B.M. et al., 2008, ApJ, 684, 888

Poggianti, B.M. et al., 2009, ApJ, astro-ph 0811.0252

Postman, M. et al., 2005, ApJ, 623, 721

Sanchez-Blazquez, P., et al. 2009, A\&A accepted

Smith, G.P., Treu. T., Ellis, R.S., Moran, S.M., Dressler, A., 2005, ApJ, 620, 78

Treu, T., Ellis, R.S., Kneib, J.-P., Dressler, A., Smail, I., Czoske, O., Oemler,

A., Natarajan, P., 2003, ApJ, 591, 53

Valentinuzzi, T. et al., 2009, A\&A in press

van der Wel, A., Holden, B.P., Franx, M., Illingworth, G.D., Postman, M.P., Kelson, D.D., Labbe', I., Wuyts, S., Blakeslee, J.P., Ford, H.C., 2007, ApJ, 670, 206

van der Wel, A., 2008, ApJL, 675, L13

Varela, J., et al., 2009, A\&A in press

Whitmore, B.C., Gilmore, D.M., Jones, C., 1993, ApJ, 407, 489

Wilman, D.J., Balogh, M.L., Bower, R.G., Mulchaey, J.S., Oemler, A., Carlberg, R.G., Morris, S.L., Whitaker, R.J., 2005, MNRAS, 358, 71

Wilman, D., Oemler, A., Mulchaey, J.S., McGee, S.L., Balogh, M.L., Bower, R.G., 2009, ApJ in press (astro-ph 0811.4425) 


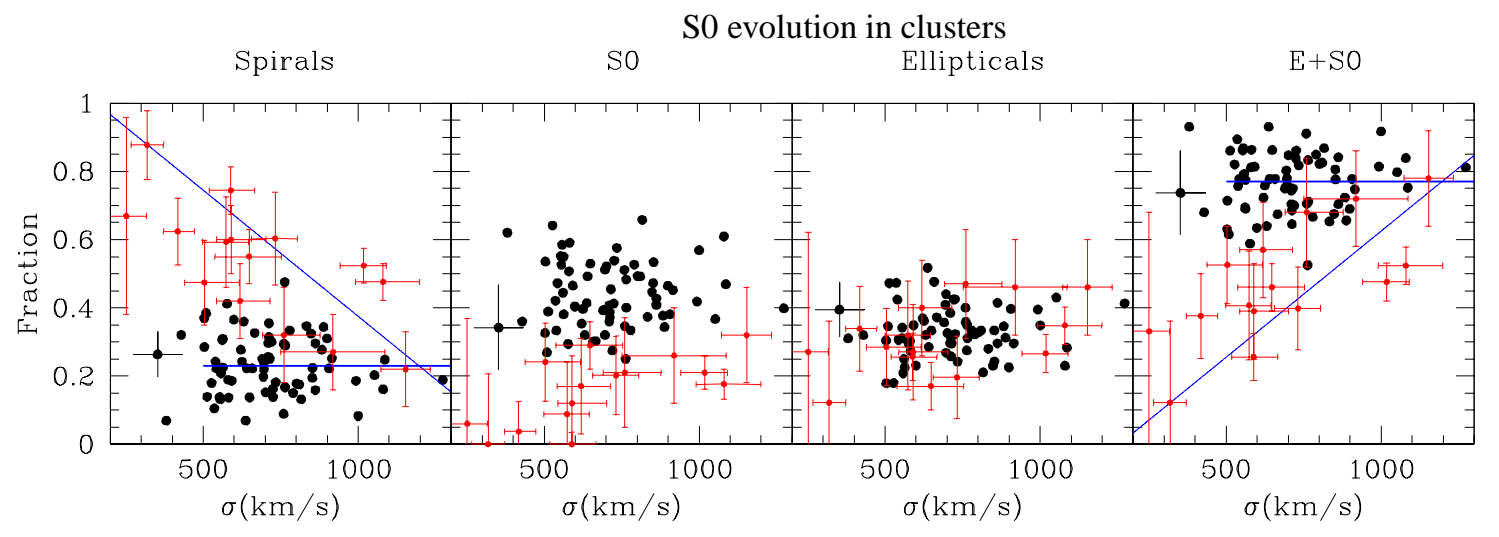

FIG. 1.- Morphological fractions within $0.6 R_{200}$ versus rest frame cluster velocity dispersion for galaxies with a passively evolving absolute magnitude limit $M_{V}<-19.5$ at the WINGS redshift. Nearby clusters (WINGS) are black circles. Average Poissonian errorbars for WINGS are shown in each panel on the leftmost datapoint. Distant clusters (EDisCS+Postman) are red circles with individual errorbars. None of the WINGS fractions is statistically correlated with $\sigma$. Solid blue lines in the leftmost panel represent the average fractions of emission-line galaxies at high-z (inclined line, EDisCS) and low-z (horizontal line, SDSS cluster sample) from Poggianti et al. (2006). Similarly, the solid blue lines in the rightmost panel are the average fractions of passively evolving (non-emission-line) galaxies.

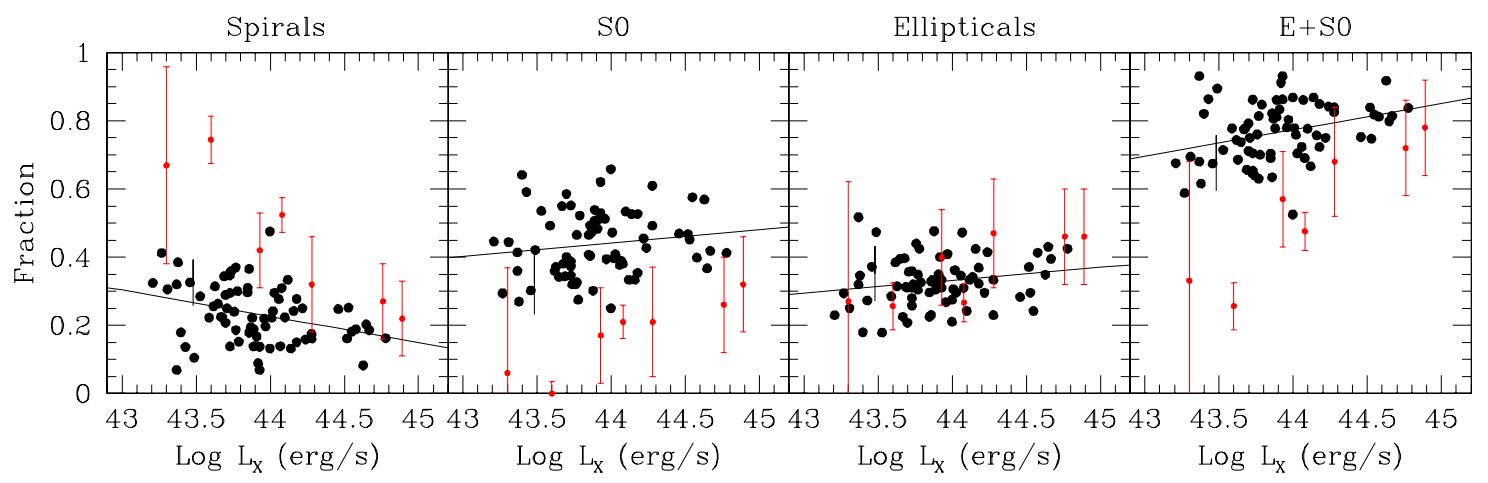

FIG. 2.- Morphological fractions versus cluster X-ray luminosity. Nearby clusters (WINGS) are black circles, with errors on fractions as in Fig. 1. Distant clusters (EDisCS+Postman with available $L_{X}$ ) are red circles. The WINGS spiral fraction is statistically anticorrelated with $L_{X}(99.6 \%)$. The least square fit is shown in each panel as a solid line. 